\title{
Influence of Paired Stimulation on the Electro- mechanical Dissociation During Low Calcium Perfusion of Isolated Rat Hearts
}

\author{
E. S. Nieuwendijk, F. L. Meijler, and D. Durrer \\ From the University Department of Cardiology and Clinical Physiology, \\ Wilhelmina-Gasthuis, Amsterdam, the Netherlands
}

AUTHORS' SyNOPSIS. During electro-mechanical dissociation in isolated rat hearts caused by low calcium perfusion, paired stimulation restored the contractions. The main factor determining the size of these contractions is the duration of the intercal between the two stimuli forming a pair.

The occurrence of an electro-mechanical dissociation during calcium-free perfusion of mammalian myocardial tissue (Mines, 1913; Cousy and Noyons, (1923) demonstrates the dominant role of calcium in the excitation-contraction coupling (Sandow, 1952). The use of radioactive calcium for the study of this excitation-contraction coupling has revealed that almost any augmentation of the size of contractions of the heart, originated by greatly differing interventions, in all instances correlates with a greater calcium influx per beat. This is, for instance, the case when cardiac glycosides, catecholamines, or calcium ions are applied, or when the frequency of contraction is increased (Winegrad, 1961; Grossman and Furchgott, 1964).

The decrease of the contractions caused by lowering the calcium concentration of the perfusion fluid, correlates with a diminished calcium influx per contraction (Grossman and Furchgott, 1964).

Another intervention giving rise to a strong potentiation of the contractions of the heart is paired stimulation (Meijler and Durrer. 1965). This effect appears to be very pronounced during low calcium perfusion (Nieuwendijk, 1966). In this study we have investigated the influence of paired stimulation on the size of the contractions at very low extraceilular calcium concentrations and compared the results with the effect of single stimulation at calcium concentrations varying between 0.3 and 8.0 m-iquiv/l.

Received April 26, 1967

\section{Methods}

Hearts of white rats. weighing approximately $250 \mathrm{~g}$, were isolated and perfused according to the method of Langendorff (1895) with a modified Tyrode solution, with a calcium concentration of 2.6 m-equiv/l. The composition of this perfusion fluid could be varied according to the experimental situation. The vertical displacement of the apex of the heart was recorded isotonically by means of a Sanborn 7 DCDT-100 transducer, connected to a Sanborn DC preamplifier, model 350-1000. The electrical activity of the heart was recorded by means of a "unipolar" epicardial electrode, connected with a Sanborn high gain preamplifier 350-2700. Control of rate and rhythm was achieved via bipolar electrodes. stitched to the area trabecularis of the right ventricle, by means of a specially designed current-source stimulator (Nieuwendijk. 1966). In each instance the strength and duration of the single driving stimuli were respectively $1 \mathrm{~mA}$ and 1 msec. To prevent interference with propagated sinus beats the atria were removed.

Paired stimulation can be effected in two ways. Either the mechanical frequency present during regular stimulation is kept constant by doubling the number of stimuli applied per time unit, during paired stimulation, or the mechanical frequency is halved by keeping the number of stimuli constant. Every second impulse in a regular train of stimuli was gradually transferred to an earlier moment till the occurrence of contractions with optimal potentiation and fusion. Paired stimulation only causes a potentiating effect if both stimuli applied croke an electrical excitation of the heart. The effect of paired stimulation was studied 15 min after the beginning of perfusion of the fluid with the new calcium concentration. After completion of each observation, perfusion at normal calcium concentration was re- 
instituted. If the contractions were identical with previous control values, perfusion with a different calcium concentration was begun. This procedure makes it impossible to study the influence of paired stimulation during perfusion without any calcium, because of the occurrence of the "calcium paradox" (Zimmerman and Hülsmann, 1966) when calcium containing fluid is reintroduced. If the extracellular calcium concentration is not lowered beyond 0.3 m-equiv/l., this "calcium paradox" does not occur; therefore the lowest extracellular calcium concentration used was 0.3 m-equiv/l.

\section{Results}

At normal extracellular calcium concentration the effect of regular single stimulation on the contractions of the isolated heart is compared with the effect obtained by paired stimulation; the mechanical frequency is kept constant (Fig. 1A).

In Fig. 1B the same procedure is repeated during low calcium perfusion $(0.5 \mathrm{~m}$-equiv/l.) At the first arrow paired stimulation is initiated and at the second arrow stopped and single stimulation is resumed. During the single stimulation period preceding paired stimulation no mechanical activity is recorded. After the application of paired stimulation it takes about 3 beats $(1.5 \mathrm{sec})$ before the first contractions become measurable. They increase gradually $: 2$ size till after about $12 \mathrm{sec}$ a steady state is attained. After cessation of paired stimulation mechanical activity disappears in about 6 beats $(3 \mathrm{sec})$.

This experiment demonstrates that paired stimulation is capable of initiating mechanical activity under experimental conditions, where single stimulation has failed to do so. It should be realized that in this experiment it is by no means clear whether the mechanical activity, originated by paired stimulation, is caused by the specific spacing of the stimuli, by the increase of the number of electrical stimuli applied per time unit, or by both. Therefore experiments were carried out in which the number of stimuli per minute was kept constant, but only the time interval between successive first and second stimuli was varied.

Figure 2 is representative for the results of 5 experiments. First the isolated heart is stimulated with a steady state interval of $500 \mathrm{msec}$, which does not initiate any mechanical activity. Next the heart is stimulated with alternating intervals of
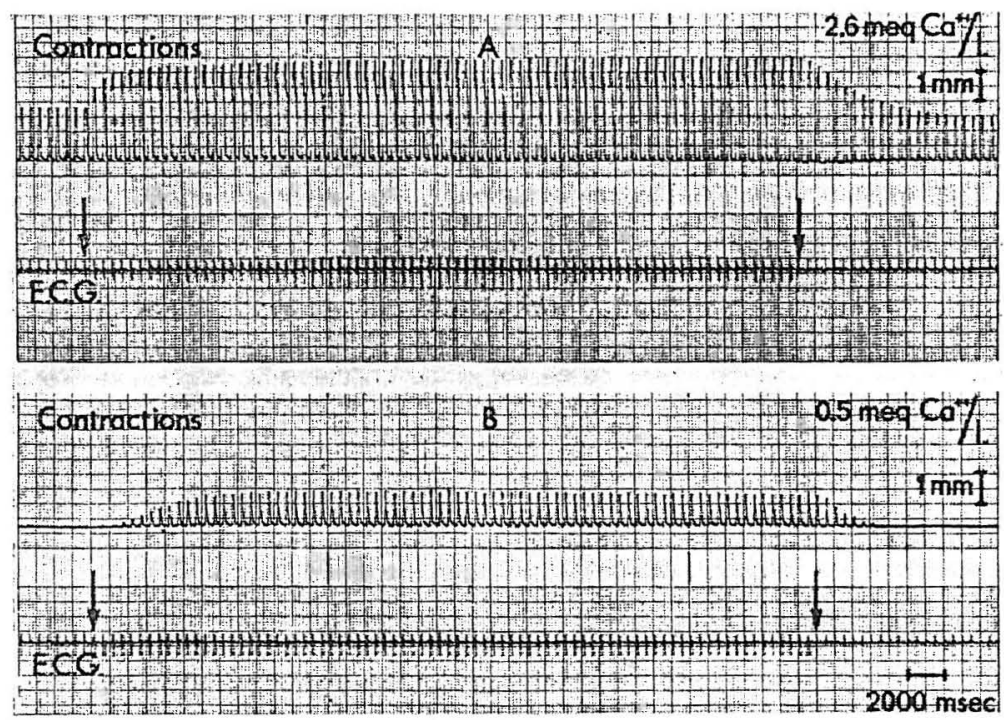

Fig. 1. After a period of regular stimulation of the heart $(120 / \mathrm{min})$, by applying stimuli every $500 \mathrm{msec}$, paired stimulation was begun and ended at the moments indicated by the first arrow and second arrow respectively. During this period of paired stimulation the duration of the intervals between the stimuli of every pair was $100 \mathrm{msec}$, the duration between the second stimulus of a pair and the first one of the following pair was $400 \mathrm{msec}$. Therefore the mechanical rate was kept constant. In row A (normal calcium concentration) the usual response to paired stimulation is seen. In row B (low calcium concentration) no mechanical activity is discernible during single stimulation. The third pair of stimuli results in a small contraction. After the beginning of paired stimulation a gradual increase in size is visible till a steady state level is reached. After stopping paired stimulation, single stimulation causes gradually decreasing contractions, till after six beats no further mechanical response is evoked. 

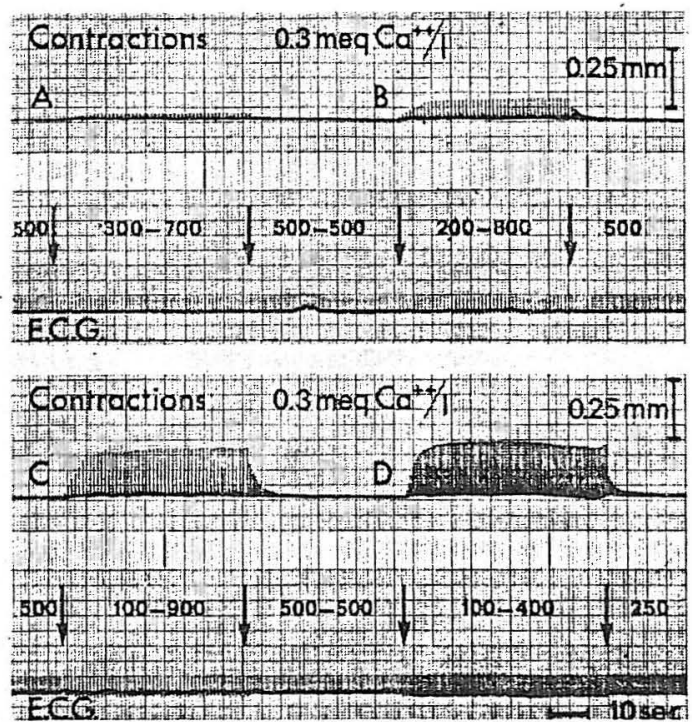

Fig. 2. In this experiment during low calcium perfusion, regular stimuli were at first applied every $500 \mathrm{msec}$, and no mechanical action was recorded. Changing of this stimulation pattern (500-500) to 300-700 (A) evoked small contractions. When, after a period of regular stimulation, this pattern was changed to 200-800 the contractions increased in size (B) and became maximal at 100-900 (C). With the latter type of stimulation maximal fusion occurred. If the electrical rate of paired stimulation were doubled (100-400) the number of contractions doubled, but the size of the contraction did not change appreciably. At the end of the experiment regular stimuli with a frequency of $4 / \mathrm{sec}(R-R$ $250 \mathrm{msec}$ ) were applied. No mechanical activity was recorded.

400 and 600 msec. Again no mechanical activity can be demonstrated. Then the stimulation pattern is changed into alternating intervals of 300 and $700 \mathrm{msec}$ (Fig. 2A), 200 and $800 \mathrm{msec}$ (Fig. 2B), and 100 and $900 \mathrm{msec}$ (Fig. 2C). Thus in all these experiments the electrical frequency is kept constant. All the stimulation patterns in Fig. 2 are separated by a period of regular stimulation with a steady state interval of 500 msec. Figure 2 (A, B, C) demonstrates that the spacing of the stimuli is of major importance for the initiation of organized mechanical activity. Even paired stimulation differing slightly from regular single stimulation may give rise to the resumption of small contraction of the isolated rat heart at calcium concentrations, where single stimulation does not cause any mechanical activity. The very important influence of the spacing between successive paired stimuli on contractile phenomena, can be made clear by doubling the number of paired stimuli-that is, changing from alternating intervals of 100 and $900 \mathrm{msec}$ to alternating intervals of 100 and $400 \mathrm{msec}$ (Fig. 2D). Only a very small increase of the contractions occurs.
During normal calcium perfusion and single stimulation such a doubling of the driving stimuli would cause a considerable increase of the contraction (Nieuwendijk, 1966). Figure 2D ends with regular stimulation with a steady state interval of $250 \mathrm{msec}$, which means a doubling of the electrical frequency in comparison with regular stimulation with a steady state interval of $500 \mathrm{msec}$. It is evident that this doubling of the frequency does not initiate any contractions. From these experiments it became clear that, at a certain extracellular calcium concentration, single stimulation cannot evoke a mechanical reaction, while paired stimulation with the same electrical frequency originates distinct contractions. In some instances with moderately lowered calcium concentration paired stimulation with relatively wide-spacing of the stimuli resulted in the recurrence of mechanical activity. In other experiments with very low calcium concentration, closer spacing was needed to evoke a similar mechanical response. This finding therefore appeared to be dependent on the calcium concentration of the perfusion fluid.

\section{Contractions $\mathrm{mm}$}

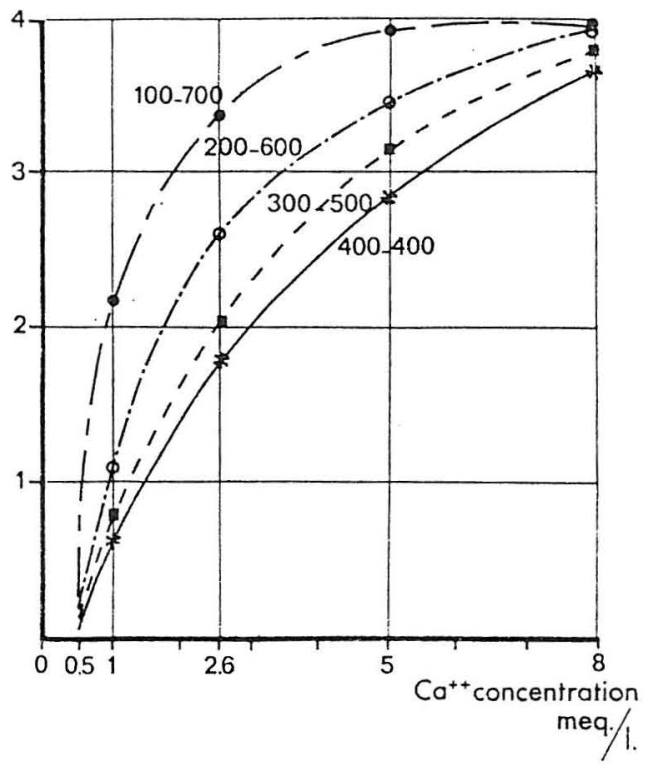

Fig. 3. The relation between the contractions of an isolated rat heart and the extracellular calcium concentration at different stimulation patterns. It is evident that at every calcium concentration paired stimulation with the closest spacing of the stimuli forming a pair resulted in the greatest shortening of the heart. This effect was very pronounced at low calcium concentrations ( 1 m-equiv/l.) and much less at concentrations higher than $5 \mathrm{~m}$-equiv/l. The intervals are given in msec. 
In a series of experiments the relation between calcium concentration of the perfusion fluid, the time interval between the first and second impulse of the paired stimuli, and the size of contractions evoked by these stimuli, was studied. In these experiments, the total number of stimuli per minute applied to the heart, was kept constant.

Isolated hearts were stimulated with the following stimulation patterns. First, with regular intervals of 400 msec. Next, every second impulse was transferred to $300 \mathrm{msec}$ after the preceding impulse, the time of occurrence of which was kept unchanged. Therefore a rhythm with alternating intervals of 300 and $500 \mathrm{msec}$ occurred. After this procedure, alternating intervals of 200 and $600 \mathrm{msec}$, followed by intervals of 100 and $700 \mathrm{msec}$, were made. This was done at extracellular calcium concentrations of $0.5,1 \cdot 0,2.6$ (normal), 5 , and 8 m-equiv/l. The contractions, measured as the amount of vertical displacement of the apex in millimetres, are plotted in Fig. 3.

From Fig. 3, representing data of such an experiment, it is clear that the depressing effect on the size of the contractions of lowering the calcium concentration, can be counteracted by decreasing the time interval between the stimuli forming a pair.

\section{Discussion}

It is by now a well-known fact that the absence of calcium ions in the perfusion fluid of isolated hearts results in an electro-mechanical dissociation.

Our experiments demonstrate that, even at the highest sensitivity of the recorder, single stimulation is not able to evoke contractions at an extracellular calcium concentration of $0.3 \mathrm{~m}$-equiv/l. Paired stimulation, however, clearly restores mechanical activity. Many authors agree that the size of the contraction in general is dependent on the number of calcium ions available for the contractile proteins during systole. For our conclusion it is not relevant to speculate about which part of these calcium ions is derived from the extracellular space, and which part from the subcellular structures.

From our experiments it can be concluded that, in comparison with single stimulation, paired stimulation is able to raise the systolic intracellular calcium concentration to such a degree that the contractile proteins can shorten. From the observation that the mechanical response becomes visible after a small number of paired stimulation cycles, it can be deduced that it takes time before this increased systolic intracellular calcium concentration is reached. The reverse situation is present after paired stimulation is changed into single stimulation.

It is also demonstrated that it is not only paired stimulation with maximal fusion that is able to increase the systolic intracellular calcium concentration, when compared with single stimulation, but also paired stimulation in which every second impulse still evokes a separable and smaller contraction.

From Fig. 2C and D it becomes clear that the shorter one of the two intervals has the greater influence on this increase of the systolic intracellular calcium concentration. For changing a rhythm with alternating intervals of 100 and $900 \mathrm{msec}$ to alternating intervals of 100 and $400 \mathrm{msec}$ does not appreciably increase the size of the recorded contractions. At zero calcium concentration paired stimulation does not give rise to any mechanical activity (Zimmerman, Meijler, Nieuwendijk, and Durrer, 1966). Thus, for the restoration of contractility by paired stimulation during this form of electro-mechanical dissociation, a minimal amount of $\mathrm{Ca}^{++}$ions in the extracellular fluid must be present. This concentration is about $0.3 \mathrm{~m}$-equiv/1

The "interchangeability" (Winegrad, 1961) of steady state frequency of contraction and extracellular calcium concentration has already been demonstrated. Our experiments suggest that a similar relationship between paired stimulation and the extracellular calcium concentration exists.

In a recent review, Nayler (1967) presents additional evidence which supports the hypothesis that calcium ions play a dominant role in the effect of interval changes on myocardial contractility.

\section{Summary}

It is demonstrated that during electro-mechanical dissociation of isolated rat hearts, perfused according to the Langendorff method with a modified Tyrode solution, with very low calcium concentration, contractions may reappear, after transposing every second impulse of a regular train of stimuli, to an earlier moment (paired stimulation).

The contractions increase in size during shortening of this distance and become optimal during paired stimulation with maximal fusion. Evidence is presented that the spacing of the two successive stimuli of each pair is of major importance in evoking this phenomenon. We assume that this stimulation pattern causes an increase of intracellular calcium concentration. The effect of increase in calcium concentration in the perfusion fluid on this relation between spacing of stimuli and contraction is compatible with this hypothesis. 


\section{References}

Cousy, R. C. and Noyons, A. K. (1923). L'irritabilité du coeur et le balancement des ions. Arch. int. Physiol., 20, 1-28.

Grossman, A. and Furchgott, R. F. (1964). The effects of external calcium concentration on the distribution and exchange of calcium in resting and beating guinea-pig auricles. J. Pharmacol. exp. Ther., 143, 107-119.

- and - (1964). The effects of frequency of stimulation and calcium concentration on $\mathrm{Ca}^{45}$ exchange and contractility of the isolated guinea pig auricles. J. Pharmacol. exp. Ther., 143, 120-130.

Langendorff, O. (1895). Untersuchungen am überlebenden Säugethierherzen. Pflügers Arch. ges. Physiol., 61, 291-330.

Meijler, F. L. and Durrer, D. (1965). Physiological and clinical aspects of paired stimulation, Bull. N.Y. Acad. Med., 41, 575-591.
Mines, G. R. (1913). On functional analysis by the action of electrolytes. J. Physiol. (Lond.), 46, 188-235.

Nayler, W. G. (1967). Calcium exchange in cardiac muscle: A basic mechanism of drug action. Am. Heart J., 73, 379-394.

Nieuwendijk, E. S. (1966). The action of calcium on the contractility of the isolated rat heart. Thesis, Amsterdam.

Sandow, A. (1952). Excitation-contraction coupling in muscular response. Yale J. Biol. Med., 25, 176-20l.

Winegrad, S. (1961). The possible role of calcium in excitation-contraction coupling of heart muscle. Circulation, 24. 523-529.

Zimmerman, A. N. E. and Hülsmann, W. C. (1966). Paradoxical influence of calcium ions on the permeability of the cell membranes of the isolated rat heart. Nature (Lond.), 211, 646-647.

—, Meijler, F. L., Nieuwendijk, E. S., and Durrer, D. Unpublished observations. 